\title{
Interactions between traditional regional determinants and socio-economic status on dietary patterns in a sample of French men
}

\author{
Anne-Elisabeth Perrin ${ }^{1}$, Jean Dallongeville ${ }^{2}$, Pierre Ducimetière ${ }^{3}$, Jean-Bernard Ruidavets ${ }^{4}$, \\ Jean-Louis Schlienger ${ }^{1}$, Dominique Arveiler ${ }^{5}$ and Chantal Simon ${ }^{1} *$ \\ ${ }^{1}$ Groupe d'Etudes en Nutrition, Service de Médecine Interne et Nutrition, Hôpital de Hautepierre, 67098 Strasbourg, France \\ ${ }^{2}$ INSERM U508, Institut Pasteur, Lille, France \\ ${ }^{3}$ INSERM U258, Hôpital Paul Brousse, Villejuif, France \\ ${ }^{4}$ INSERM U558, Département d'Epidémiologie, Faculté de Médecine, Toulouse, France \\ ${ }^{5}$ Laboratoire d'Epidémiologie et de Santé Publique, Faculté de Médecine, Strasbourg, France
}

(Received 24 May 2004 - Revised 19 August 2004 - Accepted 24 August 2004)

\begin{abstract}
The aim of the present study was to assess the respective contributions of regional and socio-economic factors to dietary pattern. We used the data from the final MONICA (MONItoring of trends and determinants in Cardiovascular disease) population survey conducted in the three French centres in 1995-7 among a representative sample of 976 men aged 45-64 years. Dietary intake was assessed using a 3-d record method. Dietary patterns were identified by a factor analysis, based on fifteen food items. An analysis of variance was then used to study their relationship with regional and socio-economic determinants. Two major dietary patterns were identified: a 'Western diet', characterized by high intakes of sugar and sweets, grains, butter, added fats, eggs, potatoes and cheese; a 'prudent diet', mainly distinguished by high intakes of fruit, vegetables, olive oil and fish and low intakes of alcohol, high-fat meat and potatoes. Strong associations were mostly observed with the 'prudent diet' pattern, with a significant relationship with region, educational and incometax levels, leisure-time physical activity and smoking status. There was also a statistically significant interaction between region and educational level $(P=0.05)$, and between region and income-tax level $(P=0.03)$, indicating that the influence of socio-economic factors is different among regions. In conclusion, these results indicate large regional and socio-economic differences in the dietary patterns of this French male population. When considering the 'prudent diet' pattern, they also suggest that traditional regional influences may now be overcome by socio-economic determinants.
\end{abstract}

Dietary patterns: Geographical determinants: Socio-economic status

The effects of dietary factors on the development of chronic diseases, especially CVD and cancer, but also obesity, have been investigated in many experimental, clinical and epidemiological studies. The traditional analytical approach in nutritional epidemiology has largely focused on individual nutrient- or foodbased analyses. However, it is often difficult to separate the specific effects of nutrients or foods because of the complexity of dietary intake and the multiple potential interactions between the components of a diet (Jacques \& Tucker, 2001). The combined effects of various nutrients and foods can be observed only when the entire eating pattern is considered. In recent years another approach, based on the identification of overall dietary patterns, has been proposed $(\mathrm{Hu}, 2002)$. The interests of such an analysis have been underlined in many recent publications focused on the role of overall dietary patterns in chronic diseases (Jacques \& Tucker, 2001; Schulze \& Hu, 2002; Kant, 2004). Analysing food consumption in terms of dietary patterns is not only interesting to examine the relationship between diet and disease risk but may also have implications from a public health perspective. Identification of such patterns provides useful information to set up health and nutrition policies and guidelines for healthy food consumption.

Geographical factors are usually considered as strong determinants of dietary patterns; one of the most obvious examples of that influence is the 'Mediterranean diet', adopted by the different populations around the Mediterranean Sea (Keys et al. 1986). Regional differences have also been found in France, as shown in the first French MONICA (MONItoring of trends and determinants in Cardiovascular disease) population survey (Jost et al. 1990). People living in the south of France adopted the 'Mediterranean diet' rich in fruit and vegetables, fish, olive oil and wine, whereas in the northern regions, the traditional diet was characterized by high intakes of sausages and ham, butter, eggs, potatoes and beer.

However, some studies have suggested an attenuation of geographical differences in dietary behaviours (Huijbregts et al. 1997) and the strong contribution of socio-economic factors (Marmot et al. 1991; Roos et al. 1996; Margetts et al. 1998; Johansson et al. 1999) that may modulate geographical influences. Although many studies have explored dietary patterns 
in relation to their determinants, very few investigations have focused on the extent to which these different determinants interact. Using the data from the final French MONICA population survey, we attempted to identify dietary patterns in a random sample of nearly 1000 middle-aged men living in France, in three distinct regions characterized by traditionally different dietary habits, and to evaluate the relative contribution of the regional and socio-economic factors to the main dietary patterns.

\section{Methods}

\section{Study design and sample}

The study is based on data from the final MONICA population survey, conducted in 1995-7 in three French centres: the Urban Community of Lille in the north $(\mathrm{N})$, the department of BasRhin in the north-east (NE) and the department of Haute-Garonne in the south-west (SW) of France. Study design and data collection have been described elsewhere (WHO MONICA Project Principal Investigators, 1988). The random sample, stratified by town size, was obtained from the electoral rolls. It included 3508 men and women aged 35-64 years (1195 in N, 1131 in $\mathrm{NE}$ and 1182 in SW). The mean participation was $58 \%$.

A standard questionnaire was submitted to the participants in order to identify their socio-demographic, lifestyle and health characteristics. This questionnaire included questions on socioeconomic factors, health, physical activity, smoking and drinking habits, attitudes and knowledge concerning several diseases. Physical measurements and a venous blood sample were also performed by a specially trained nurse, following the recommendations of the WHO MONICA Manual (World Health Organization, 1986).

According to the MONICA-France study design, the nutritional study was carried out only in a subsample of men, comprising 976 subjects ( 376 in N, 264 in NE and 336 in SW), aged 4564 years. Food intake data were collected by trained dietitians, using a prospective 3-d food intake record. The data were carefully checked and portions were estimated with a validated set of photographs.

\section{Study variables}

The statistical analyses were carried out using the following dietary, regional, socio-economic and lifestyle variables.

Indicators of dietary habits. Fifteen foods or food groups collected by the 3-d food intake record were included in the analysis to describe eating patterns. These were: (1) grains: bread and cereal; (2) low-fat (LF) dairy products: milk and yoghurt; (3) cheese; (4) eggs; (5) LF meat, with less than $10 \%$ fat (including poultry, beef, veal); (6) high-fat (HF) meat, with more than $10 \%$ fat (including sausages, fat pork meat, lamb); (7) fish; (8) fruit; (9) vegetables; (10) potatoes; (11) sugar and sweets; (12) butter; (13) alcohol (including beer, wine and liquor); (14) added fats; (15) olive oil. All food items were expressed as mean intake in $\mathrm{g} / \mathrm{d}$.

Geographical variable. The geographical variable was the MONICA centre, in three categories: N; NE; SW.

Socio-economic variables. Socio-economic variables were the educational and income-tax levels. Educational level was divided into three groups: primary school; secondary school; technical training/university. Income-tax level was classified in three categories as: no and low income tax $(<750$ euros/year); medium income tax (750-2300 euros/year); high income tax $(>2300$ euros/year).
Lifestyle indicators. Lifestyle indicators were smoking status and level of leisure-time physical activity (LPA). Smoking status was classified as no current smoking or current smoking. LPA was categorized as no LPA, moderate LPA almost every week, intensive LPA for at least 20 min once or twice a week, intensive LPA for at least $20 \mathrm{~min}$ three times or more per week.

\section{Statistical analyses}

Statistical analyses were performed with the Statistical Analysis Systems statistical software package version 8.2 (SAS Institute, Cary, NC, USA). Dietary patterns were identified by a factor analysis, a multivariate statistical technique that uses dietary information (from food frequency questionnaires or dietary records) to identify common underlying dimensions (factor or patterns) of food consumption. This technique aggregates specific food items on the basis of their degree of correlation $(\mathrm{Hu}, 2002)$. We conducted the analyses with the SAS System PROC FACTOR procedure: we used a principal component analysis to extract the initial factors and an orthogonal transformation (Varimax rotation function in SAS) to make it simpler and easier to interpret factors. To identify the number of factors to be retained, we considered eigenvalue $>1 \cdot 0$, the scree test and the interpretability of the factors. A diagram of Cronbach's coefficient $\alpha$ was also used to test the pattern structure and to determine the food items contributing to the factor. Food items with absolute factor loadings $>0.25$ were considered as significantly contributing to the pattern. Factor scores (i.e. the individual values of the factor) for each pattern were then derived by summing observed intakes of the component food items weighted by factor loadings. These scores were subsequently used in an ANOVA to characterize the dietary patterns across the regional, socio-economic and lifestyle determinants. Furthermore, ANOVA was used to test the effects on dietary patterns of region $x$ educational level and of region $\times$ income-tax level interactions. Results were expressed as means with their standard errors and differences in dietary patterns were regarded as significant when the $P$ value was $<0 \cdot 05$.

In order to minimize reporting errors, the analyses were done with and without taking into account underreporters. We identified underreporters from the ratio of daily energy intake to theoretical BMR, estimated using the Schofield equations based upon weight, age and sex (Schofield et al. 1985). The underreporting cut-off value was based on estimation of the lowest physically plausible energy intake for weight maintenance; in this study based on a 3-d record, the cut-off value was 1.05 , as proposed by Goldberg (Goldberg et al. 1991).

\section{Results}

\section{Socio-economic and lifestyle characteristics}

Table 1 shows the regional, socio-economic and lifestyle characteristics of the population under study. The average age of the 976 participants was $55 \cdot 1$ (SD 6.1) years with 488 men aged $45-54$ years and 488 men aged 55-64 years. The subjects had predominantly received secondary or technical education and the majority paid a medium or high income tax $21 \%$ were current smokers. A moderate LPA was reported by the majority of the subjects $(49.3 \%)$. About $21 \%$ were obese $\left(\mathrm{BMI} \geq 30 \mathrm{~kg} / \mathrm{m}^{2}\right)$ and almost $50 \%$ were overweight (BMI $25-29.9 \mathrm{~kg} / \mathrm{m}^{2}$ ). 
Table 1. Regional, socio-economic and lifestyle characteristics of the 976 participants

\begin{tabular}{|c|c|c|}
\hline & $n$ & $\%$ \\
\hline \multicolumn{3}{|l|}{ Age in years } \\
\hline mean & $55 \cdot 1$ & \\
\hline SD & $6 \cdot 1$ & \\
\hline \multicolumn{3}{|l|}{ Region (MONICA centre) } \\
\hline North & 376 & 38.5 \\
\hline North-east & 264 & $27 \cdot 0$ \\
\hline South-west & 336 & $34 \cdot 4$ \\
\hline \multicolumn{3}{|l|}{ Educational level } \\
\hline Primary & 225 & $23 \cdot 1$ \\
\hline Secondary or technical & 529 & $54 \cdot 2$ \\
\hline University & 222 & $22 \cdot 7$ \\
\hline \multicolumn{3}{|l|}{ Income-tax level } \\
\hline None and low & 309 & $32 \cdot 3$ \\
\hline Medium & 329 & 34.3 \\
\hline High & 320 & 33.4 \\
\hline \multicolumn{3}{|l|}{ Smoking status } \\
\hline No current smoking & 766 & 78.5 \\
\hline Current smoking & 210 & $21 \cdot 5$ \\
\hline \multicolumn{3}{|l|}{ Physical activity } \\
\hline No LPA & 178 & $18 \cdot 3$ \\
\hline Moderate LPA & 480 & $49 \cdot 3$ \\
\hline Intensive LPA 1-2 times per week & 179 & $18 \cdot 4$ \\
\hline Intensive LPA $\geq 3$ times per week & 137 & $14 \cdot 1$ \\
\hline \multicolumn{3}{|l|}{$\mathrm{BMI}$} \\
\hline$<25 \mathrm{~kg} / \mathrm{m}^{2}$ & 286 & 29.5 \\
\hline $25-30 \mathrm{~kg} / \mathrm{m}^{2}$ & 484 & 49.9 \\
\hline$\geq 30 \mathrm{~kg} / \mathrm{m}^{2}$ & 199 & $20 \cdot 6$ \\
\hline
\end{tabular}

LPA, leisure-time physical activity.

\section{Dietary patterns}

Factor analysis extracted two major factors from the fifteen foods or food groups collected by the $3-\mathrm{d}$ record. These factors accounted for $26.7 \%$ of the total variance in food intake. The factor loading matrix is shown in Table 2. Factor 1 (termed 'Western diet') explained $13.6 \%$ of the total variance. It was positively and heavily associated with the following foods or food groups: sugar and sweets, grains, butter, added fats, eggs, dairy products, potatoes, cheese and fruit. Factor 2 (termed 'prudent diet'), accounting for

Table 2. Factor loading matrix for the major factors (diet patterns) in the final French MONICA survey (976 men)*

\begin{tabular}{lcc}
\hline & Factor 1 & Factor 2 \\
\hline Sugar and sweets & 0.70 & - \\
Grains & 0.54 & - \\
Butter & 0.50 & - \\
Added fats & 0.44 & - \\
Eggs & 0.43 & - \\
Dairy products & 0.37 & 0.34 \\
Cheese & 0.26 & - \\
Fruit & 0.26 & 0.56 \\
Vegetables & - & 0.54 \\
Olive oil & - & 0.48 \\
Fish & - & 0.32 \\
Alcohol & - & -0.44 \\
High-fat meat & - & -0.50 \\
Potatoes & 0.33 & -0.55 \\
\hline
\end{tabular}

* Food items with absolute factor loadings $>0.25$ were considered as significantly contributing to a pattern and are represented in the table. A positive loading indicates a positive association with the factor, whereas a negative loading indicates an inverse association. High loadings indicate strong associations between the corresponding observed variables and patterns.
$13.1 \%$ of the variance, was positively associated with fruit, vegetables, olive oil, dairy products and fish, and negatively associated with alcohol, HF meat and potatoes. The variable LF meat, which had a very low loading, was not assigned to any factor. However, as we considered that LF meat could not be ignored, its $z$-score was included as a separate 'factor' in the subsequent analysis.

\section{Geographical, socio-economic and lifestyle factors associated with the dietary patterns}

The univariate associations of the two major dietary patterns and of LF meat with regional, socio-economic and lifestyle determinants were examined by ANOVA. The results, summarized by the factor score for factor 1 and factor 2 and the $z$-score for LF meat, are presented in Table 3.

The first dietary pattern was significantly associated with the region $(P<0.01)$, men living in NE being more likely to adhere to this pattern, but not with socio-economic factors. The only significant association with lifestyle factors was found for physical activity $(P=0 \cdot 04)$.

All the studied regional, socio-economic and lifestyle variables were associated with the second dietary pattern. A strong association was observed with the region $\left(P<10^{-4}\right)$ : the 'prudent diet' was mostly favoured by men living in SW. Educational level $\left(P<10^{-4}\right)$ and income-tax level $\left(P<10^{-3}\right)$ also had a significant effect on this pattern, with a better adherence from people with a higher educational level and with medium or high income-tax levels. There was a significant and positive association with physical activity $\left(P<10^{-4}\right)$ : men who had regular physical activity favoured the 'prudent diet'. Conversely, a negative association was observed with smoking status $\left(P<10^{-4}\right)$ : smokers were less likely to follow that pattern.

The only significant association for LF meat was observed with the region $(P<0 \cdot 01)$, men from SW reporting the highest LF meat intake.

Relative contribution of regional and educational factors to the dietary patterns

In order to evaluate the relative influence of regional and socioeconomic factors on the dietary patterns, the ANOVA was repeated with an interaction factor: region $\times$ educational level. We found no significant interaction either for the 'Western diet' pattern $(P=0.75)$ or for LF meat $(P=0.63)$. Region $\times$ educational level interaction was, however, significant $(P=0.05)$ for the 'prudent diet' pattern, indicating that the contribution of these two determinants was not independent. Results are detailed in Table 4. A significant effect of the region was observed for each educational level, but was stronger for the primary and the secondary educational levels $\left(P<10^{-4}\right)$ than for the university educational level $(P=0.03)$. The effect of the educational level determinant was significant in the NE and $\mathrm{N}\left(P<10^{-4}\right)$, but not in the SW. A significant interaction was also observed for the 'prudent diet' $(P=0.03)$, when the interaction factor region $\times$ income-tax level was considered in the ANOVA (Table 5). Seen alternatively, the prudent diet score was high in the SW, even among those with little education or low income; this was not the case in the $\mathrm{N}$ and $\mathrm{NE}$.

None of these results was modified by the exclusion of (1) underreporters or (2) subjects reporting being on a diet for medical reason. 
Table 3. Mean factor score and significance values from analysis of variance of food factors by region, educational level, income-tax level, smoking status and physical activity level

\begin{tabular}{|c|c|c|c|c|c|c|c|c|c|}
\hline & \multicolumn{3}{|c|}{ Factor 1 'Western diet' } & \multicolumn{3}{|c|}{ Factor 2 'prudent diet' } & \multicolumn{3}{|c|}{ Low-fat meat (z-score) } \\
\hline & Mean & SE & $P$ value & Mean & SE & $P$ value & Mean & SE & $P$ value \\
\hline North & -0.097 & 0.051 & & -0.298 & 0.049 & & -0.138 & 0.051 & \\
\hline North-east & 0.178 & 0.061 & & -0.097 & 0.058 & & 0.041 & 0.061 & \\
\hline South-west & -0.031 & 0.054 & $<0.01$ & 0.409 & 0.052 & $<10^{-4}$ & 0.122 & 0.054 & $<0.01$ \\
\hline \multicolumn{10}{|l|}{ Educational level } \\
\hline Secondary or technical & -0.007 & 0.043 & & -0.056 & 0.042 & & 0.023 & 0.043 & \\
\hline University & -0.093 & 0.067 & $0 \cdot 10$ & 0.383 & 0.066 & $<10^{-4}$ & 0.015 & 0.067 & 0.49 \\
\hline \multicolumn{10}{|l|}{ Income-tax level } \\
\hline No and low income-tax level & 0.009 & 0.056 & & -0.195 & 0.056 & & -0.026 & 0.057 & \\
\hline Medium income-tax level & -0.025 & 0.054 & & 0.077 & 0.055 & & 0.095 & 0.055 & \\
\hline High income-tax level & -0.008 & 0.055 & 0.91 & $0 \cdot 108$ & 0.055 & $<10^{-3}$ & -0.052 & 0.056 & 0.14 \\
\hline \multicolumn{10}{|l|}{ Smoking status } \\
\hline No current smoking & 0.001 & 0.036 & & 0.122 & 0.035 & & 0.009 & 0.036 & \\
\hline \multicolumn{10}{|l|}{ Physical activity (LPA) } \\
\hline No LPA & -0.133 & 0.075 & & -0.217 & 0.074 & & -0.108 & 0.075 & \\
\hline Moderate LPA & -0.009 & 0.046 & & -0.093 & 0.045 & & 0.012 & 0.045 & \\
\hline Intensive LPA 1-2 times per week & 0.167 & 0.075 & & 0.157 & 0.073 & & 0.021 & 0.075 & \\
\hline Intensive LPA $\geq 3$ times per week & -0.016 & 0.085 & 0.04 & 0.406 & 0.084 & $<10^{-4}$ & 0.052 & 0.085 & 0.46 \\
\hline
\end{tabular}

LPA, leisure-time physical activity.

Table 4. Mean factor score and significance values from analysis of variance of factor 2 ('prudent diet') by region for the three educational levels and by educational level in the three regions

\begin{tabular}{|c|c|c|c|c|c|c|c|}
\hline \multirow[b]{3}{*}{ Educational levels } & \multicolumn{6}{|c|}{ Region } & \multirow[b]{3}{*}{$P$} \\
\hline & \multicolumn{2}{|c|}{ North } & \multicolumn{2}{|c|}{ North-east } & \multicolumn{2}{|c|}{ South-west } & \\
\hline & Mean & SE & Mean & SE & Mean & SE & \\
\hline Primary & -0.497 & 0.082 & -0.517 & 0.170 & 0.344 & 0.114 & $<10^{-4}$ \\
\hline Secondary & -0.372 & 0.071 & -0.166 & 0.071 & 0.357 & 0.069 & $<10^{-4}$ \\
\hline University & 0.221 & 0.108 & 0.315 & 0.120 & 0.567 & 0.099 & 0.03 \\
\hline$P$ value & \multicolumn{2}{|c|}{$<10^{-4}$} & \multicolumn{2}{|c|}{$<10^{-4}$} & \multicolumn{2}{|c|}{0.20} & \\
\hline
\end{tabular}

Table 5. Mean factor score and significance values from analysis of variance of factor 2 ('prudent diet') by region for the three income-tax levels and by income-tax level in the three regions

\begin{tabular}{|c|c|c|c|c|c|c|c|}
\hline \multirow[b]{3}{*}{ Income-tax levels } & \multicolumn{6}{|c|}{ Region } & \multirow[b]{3}{*}{$P$} \\
\hline & \multicolumn{2}{|c|}{ North } & \multicolumn{2}{|c|}{ North-east } & \multicolumn{2}{|c|}{ South-west } & \\
\hline & Mean & SE & Mean & SE & Mean & SE & \\
\hline None or low & -0.501 & 0.074 & -0.219 & 0.103 & 0.352 & 0.104 & $<10^{-4}$ \\
\hline Medium & -0.314 & 0.086 & -0.057 & 0.109 & 0.503 & 0.086 & $<10^{-4}$ \\
\hline High & -0.017 & 0.09 & -0.005 & 0.096 & 0.335 & 0.09 & 0.01 \\
\hline$P$ value & \multicolumn{2}{|c|}{$<10^{-3}$} & \multicolumn{2}{|c|}{0.28} & 0.33 & & \\
\hline
\end{tabular}

\section{Discussion}

In the present study, we identified two main dietary patterns in a large representative sample of French men and evaluated their association with regional, socio-economic and lifestyle determinants. The first dietary pattern, named 'Western diet', was characterized by a higher intake of sugar and sweets, grains, butter, added fats, eggs, dairy products, potatoes, cheese and fruit. The second pattern, qualified as 'prudent diet', was rich in fruit, vegetables, olive oil, dairy products and fish, and poor in alcohol, HF meat and potatoes. Some food groups (dairy products, fruit and potatoes) loaded on different patterns simultaneously. However, although dairy products loaded comparably on both patterns and cannot therefore be assigned to a particular pattern, the loading of fruit indicates a greater contribution to the 'prudent diet' pattern. For potatoes, a positive association was observed with the 'Western diet' pattern, whereas it was negative with the 'prudent diet' pattern. 
The 'Western diet' pattern was influenced by regional but not by socio-economic factors, and no interaction between region and educational level and between region and income-tax level was found for this pattern. By contrast, both regional and socio-economic factors contributed to the 'prudent diet' pattern with, moreover, a positive interaction between region and educational level and between region and income-tax level, suggesting that regional influences are modulated by socio-economic influences in the $\mathrm{N}$ and NE.

In this sample of French men, the 'Western diet' and 'prudent diet' patterns derived from factor analysis were much like those observed in several previous studies using the same analysis. The terms 'prudent' and 'Western' dietary patterns were first used by Slattery in 1998, in a case-control study examining associations between dietary eating patterns and the risk of colon cancer. Six dietary patterns were generated by the factor analysis, among which were a 'Western pattern', characterized by a higher intake of red meat, processed meat, fast food, refined grains and sugar-containing foods, and a 'prudent pattern', characterized by a higher intake of fruit, vegetables, fish and poultry (Slattery et al. 1998). Using the data from two large cohort studies, the Nurses' Health Study and the Health Professionals' Follow-up Study, Hu also derived prudent and Western patterns, and then examined the relationship between these major eating patterns and the risk of CHD (Hu et al. 2000; Fung et al. 2001a), type 2 diabetes (van Dam et al. 2002) and biochemical markers of obesity and CVD risk (Fung et al. 2001b). A protective effect of the 'prudent diet' was demonstrated in all these studies.

Although similarities might exist between the present study and these studies of US populations, as well as some studies of European populations (Osler et al. 2001, 2002; Schulze et al. 2001), some differences can be evidenced. However, factor analysis is always data-driven, thus defining population-specific patterns. The results are likely to represent patterns that are in some aspects unique to the French population and the current study findings may therefore be limited to this population.

Geographical variations across Europe have been investigated in many studies comparing dietary patterns between northern and southern European populations (Evans et al. 1995; Holdsworth et al. 2000). A north-south gradient in diet quality has been described, with a higher intake of energy-dense foods by northern European populations, whereas southern populations are more likely to adhere to a Mediterranean-style diet, mainly characterized by a large intake of fruit and vegetables, fish and olive oil. This north-south gradient was also observed in France in the first MONICA population survey, conducted in 1985-7, which underlined the major effect of region (Jost et al. 1990; Perrin et al. 2002). Results from this earlier study showed important variations in eating habits between the three centres, which persist in the final study. The main differences in the 1985-7 study concerned the consumption of LF meat, fruit and vegetables, bread, olive oil and wine, privileged by the inhabitants of the south-west. Most of these foods were components of the 'prudent diet', still favoured in this southern region. Moreover, in this first MONICA study, the influence of the socio-economic status appeared less important than the effect of the region. Only slight differences were noted between the educational levels, with a significant higher consumption of fruit among highly educated people, and of potatoes, bread and added fats among lower-educated people.

As documented in several previous studies (Smith \& Baghurst, 1992; Roos et al. 1996; Hjartaker \& Lund, 1998; Margetts et al.
1998; Johansson et al. 1999), we found that dietary habits differed between low and high social status groups. Studies on the influence of socio-economic differences on dietary patterns usually report a higher intake of fruit and vegetables in people with a higher socio-economic status, which is in accordance with our results. These socio-economic differences also concerned other behaviours, like smoking or LPA. Indeed, healthy dietary habits were mainly associated with other healthy lifestyle factors. Thus, the concept of 'prudent diet' could be extended to a 'prudent profile' that includes no smoking, low alcohol consumption and LPA, which are more frequently observed in high-status groups. For several authors, the distribution of this healthy behaviour, including dietary habits, may explain differences in mortality and morbidity between social classes (Marmot et al. 1991; Lynch et al. 1996).

The main finding of the present study was the demonstration that relationships between diet and regional determinants differed according to socio-economic influences (and conversely). The influence of region was mainly observed among people of low and medium socio-economic levels, who seemed to favour their traditional region's diet. This influence was less significant among the subjects of university and high income-tax levels, who are more likely to adhere to the 'prudent diet', whatever their region of origin. Yet, in south-west France, where a Mediterranean-style diet is favoured, the effect of educational level and of income-tax level was not significant. Tradition and lower cost of healthy foods (especially of fruit and vegetables) seemed, working together, to counteract the relevance of socio-economic status in the SW region.

There are some limitations to the present study. The first one concerns the food data collection. Dietary surveys were based on a record method, which appears well adapted to evaluate total energy and macronutrient intake but is less likely to be representative of long-term dietary habits. However, the survey was carried out over a whole year, so that all seasons were equally represented. Moreover, in the MONICA centre of NE, where a food frequency questionnaire was also used to determine dietary intake, we showed that data obtained by the 3-d record and the food frequency questionnaire correlate well with regard to individual food items (unpublished results).

The second limitation could apply to all studies using a factor analysis to identify dietary patterns and has already been discussed (Martinez et al. 1998; Hu et al. 2000; Schulze et al. 2001). Results of factor analysis are strongly affected by subjective analytic but important decisions, including the assignment of food items into food groups, the number of factors to extract, the method of rotation and even the labelling of components. Finally, the two major patterns accounted for only $26 \%$ of the total variance, which is in agreement with most previous studies using factor analysis. As the extracted patterns account only for a moderate part of the total variance, the potential existence of other patterns may be discussed. However, the percentage of variance explained by the factors should be interpreted with caution, because it largely depends on the total number of variables included in the analysis and on the degree to which these variables are reduced.

In conclusion, this study identified in a French population two main dietary patterns, opposed in terms of nutritional quality, and indicate large regional and socio-economic differences in these patterns. Our main finding was the demonstration that the contribution of regional and socio-economic factors was not 
independent. Our results suggest that a low socio-economic status population is at relatively higher risk of diet-related health problems in regions where the traditional diet is of low quality, thus identifying a population potentially in special need of attention from public health agencies.

\section{Acknowledgements}

The WHO-MONICA population survey developed in France was supported by grants from the Conseil Régional du Nord-Pas de Calais, the Caisse Primaire d'Assurance Maladie de Sélestat, the Association Régionale de Cardiologie d'Alsace, ONIVINS, Parke-Davis Laboratory, the Mutuelle Générale de l'Education Nationale (MGEN), the Réseau National de Santé Publique, the Direction Générale de la Santé, the Institut National de la Santé Et de la Recherche Médicale (INSERM), the Institut Pasteur de Lille and the Unité d'Evaluation du Centre Hospitalier et Universitaire de Lille.

\section{References}

Evans AE, Ruidavets JB, McCrum EE, et al. (1995) Autres pays, autres coeurs? Dietary patterns, risk factors and ischaemic heart disease in Belfast and Toulouse. QJM 88, 469-477.

Fung TT, Rimm EB, Spiegelman D, Rifai N, Tofler GH, Willett WC \& Hu FB (2001b) Association between dietary patterns and plasma biomarkers of obesity and cardiovascular disease risk. Am J Clin Nutr 73, 61-67.

Fung TT, Willett WC, Stampfer MJ, Manson JE \& Hu FB (2001a) Dietary patterns and the risk of coronary heart disease in women. Arch Intern Med 161, 1857-1862.

Goldberg GR, Black AE, Jebb SA, Cole TJ, Murgatroyd PR, Coward WA \& Prentice AM (1991) Critical evaluation of energy intake data using fundamental principles of energy physiology: 1. Derivation of cut-off limits to identify under-recording. Eur J Clin Nutr 45, 569-581.

Hjartaker A \& Lund E (1998) Relationship between dietary habits, age, lifestyle, and socioeconomic status among adult Norwegian women. The Norwegian Women and Cancer Study. Eur J Clin Nut 52, 565-572.

Holdsworth M, Gerber M, Haslam C, Scali J, Beardsworth A, Avallone MH \& Sherratt E (2000) A comparison of dietary behaviour in Central England and a French Mediterranean region. Eur J Clin Nutr 54, 530-539.

Hu FB (2002) Dietary patterns analysis: a new direction in nutritional epidemiology. Curr Opin Lipidol 13, 3-9.

Hu FB, Rimm EB, Stampfer MJ, Ascherio A, Spiegelman D \& Willett WC (2000) Prospective study of major dietary patterns and risk of coronary heart disease in men. Am J Clin Nutr 72, 912-921.

Huijbregts P, Feskens E, Rasanen L, Fidanza F, Nissinen A, Menotti A \& Kromhout D (1997) Dietary pattern and 20 year mortality in elderly men in Finland, Italy, and The Netherlands: longitudinal cohort study. BMJ 315, 13-17.

Jacques PF \& Tucker KL (2001) Are dietary patterns useful for understanding the role of diet in chronic diseases? Am J Clin Nutr 73, 1-2.

Johansson L, Thelle DS, Solvoll K, Bjornoboe GE \& Drevon CA (1999) Healthy dietary habits in relation to social determinants and lifestyle factors. Br J Nutr 81, 211-220.
Jost JP, Simon C, Nuttens MC, Bingham A, Ruidavets JB, Cambou JP, Arveiler D, Lecerf JM, Schlienger JL \& Douste-Blazy P (1990) Comparison of dietary patterns between population samples in the three French MONICA nutritional surveys. Rev Epidemiol Sante Publique 38, 517-523.

Kant AK (2004) Dietary patterns and health outcomes. J Am Diet Assoc 104, 615-635.

Keys A, Menotti A, Karvonen MJ, et al. (1986) The diet and 15-year death rate in the seven countries study. Am J Epidemiol 124, 903-915.

Lynch JW, Kaplan GA, Cohen RD, Tuomilehto J \& Salonen JT (1996) Do cardiovascular risk factors explain the relation between socioeconomic status, risk of all-cause mortality, cardiovascular mortality, and acute myocardial infarction? Am J Epidemiol 144, 934-942.

Margetts BM, Thompson RL, Speller V \& McVey D (1998) Factors which influence 'healthy' eating patterns: results from the 1993 Health Education Authority health and lifestyle survey in England. Public Health Nutrition 1, 193-198.

Marmot MG, Smith GD, Stansfeld S, Patel C, North F, Head J, White I, Brunner E \& Feeney A (1991) Health inequalities among British civil servants: the Whitehall II study. Lancet 337, 1387-1393.

Martinez ME, Marshall JR \& Sechrest L (1998) Invited commentary: Factor analysis and the search for objectivity. Am J Epidemiol 148, 17-19.

Osler M, Heitmann BL, Gerdes LU, Jorgensen LM \& Schroll M (2001) Dietary patterns and mortality in Danish men and women: a prospective observational study. Br J Nutr 85, 219-225.

Osler M, Helms Andreasen A, Heitmann B, Hoidrup S, Gerdes U, Morch Jorgensen L \& Schroll M (2002) Food intake patterns and risk of coronary heart disease: a prospective cohort study examining the use of traditional scoring techniques. Eur J Clin Nutr 56, 568-574.

Perrin AE, Simon C, Hedelin G, Arveiler D, Schaffer P \& Schlienger JL (2002) Ten-year trends of dietary intake in a middle-aged French population: relationship with educational level. Eur J Clin Nutr 56, 393-401.

Roos E, Prätällä R, Lahelma E, Kleemola P \& Pietinen P (1996) Modern and healthy?: socioeconomic differences in the quality of diet. Eur $J$ Clin Nutr 50, 753-760.

Schofield WN, Schofield C \& James WPT (1985) Basal metabolic rate. Hum Nutr Clin Nutr 39C, Suppl. 1, 1-96.

Schulze MB, Hoffmann K, Kroke A \& Boeing H (2001) Dietary patterns and their association with food and nutrient intake in the European Prospective Investigation into Cancer and Nutrition (EPIC)-Potsdam study. Br J Nutr 85, 363-373.

Schulze MB \& Hu FB (2002) Dietary patterns and risk of hypertension, type 2 diabetes mellitus, and coronary heart disease. Curr Atheroscler Rep 4, 462-467.

Slattery ML, Boucher KM, Caan BJ, Potter JD \& Ma KN (1998) Eating patterns and risk of colon cancer. Am J Epidemiol 148, 4-16.

Smith AM \& Baghurst KI (1992) Public health implications of dietary differences between social status and occupational category groups. $J$ Epidemiol Community Health 46, 409-416.

van Dam RM, Willett WC, Rimm EB, Stampfer MJ \& Hu FB (2002) Dietary fat and meat intake in relation to risk of type 2 diabetes in men. Diabetes Care 25, 417-424.

WHO MONICA Project Principal Investigators (1988) The World Health Organization MONICA Project (MONItoring trends and determinants in CArdiovascular disease). A major international collaboration. $J$ Clin Epidemiol 41, 105-114.

World Health Organization (1986) MONICA Manual, version 1.1. CVD/ MNC. Geneva: World Health Organization. 